\title{
On the mean values of an analytic function
}

\author{
by G. S. SRivastava and Sunita Rani (Roorkee)
}

Abstract. Let $f(z), z=r e^{i \theta}$, be analytic in the finite disc $|z|<R$. The growth properties of $f(z)$ are studied using the mean values $I_{\delta}(r)$ and the iterated mean values $N_{\delta, k}(r)$ of $f(z)$. A convexity result for the above mean values is obtained and their relative growth is studied using the order and type of $f(z)$.

1. Let $f(z)=\sum_{n=0}^{\infty} a_{n} z^{n}, z=r e^{i \theta}$, be analytic in the disc $|z|<R$, $0<R<\infty$. For $0 \leq r<R$, we set $M(r)=\max _{|z|=r}|f(z)|$. Then the order $\varrho$ and lower order $\lambda$ of $f(z)$ are defined as (see [4])

$$
\lim _{r \rightarrow R}\left\{\begin{array}{l}
\sup \frac{\log ^{+} \log ^{+} M(r)}{\operatorname{lig} x}=\left\{\begin{array}{l}
\varrho, \\
\lambda,
\end{array} \quad 0 \leq \lambda \leq \varrho \leq \infty,\right.
\end{array}\right.
$$

where $x=R r /(R-r)$ and $\log ^{+} t=\max \{0, \log t\}$. When $0<\varrho<\infty$, we define the type $T$ and lower type $\tau(0 \leq \tau \leq T \leq \infty)$ of $f(z)$ as

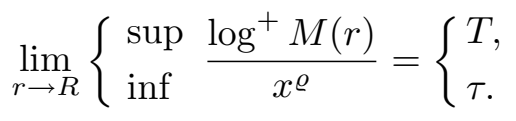

Let $m(r)=\max _{n \geq 0}\left\{\left|a_{n}\right| r^{n}\right\}$ be the maximum term in the Taylor series expansion of $f(z)$ for $|z|=r$. If $f(z)$ is of finite order $\varrho$, then ([1], [3])

$$
\log m(r) \simeq \log M(r) \quad \text { as } r \rightarrow R .
$$

Hence $m(r)$ can be used in place of $M(r)$ in (1.1) and (1.2) for defining $\varrho$, $\lambda$ etc.

The following mean value of an analytic function $f(z)$ was introduced by Hardy [2]:

$$
I_{\delta}(r)=\left[J_{\delta}(r)\right]^{1 / \delta}=\left[\frac{1}{2 \pi} \int_{0}^{2 \pi}\left|f\left(r e^{i \theta}\right)\right|^{\delta} d \theta\right]^{1 / \delta}
$$

1991 Mathematics Subject Classification: 30B10.

Key words and phrases: analytic function, maximum term, order, type, mean values. 
where $0<\delta<\infty$. We introduce the following weighted mean of $f(z)$ :

$$
N_{\delta, k}(r)=x^{-k} \int_{0}^{r} I_{\delta}(y)\left(\frac{R y}{R-y}\right)^{k+1} \frac{d y}{y^{2}},
$$

where $x=R r /(R-r)$ and $0<k<\infty$.

In this paper we have studied the growth properties of the analytic function $f(z)$ through its mean values $I_{\delta}(r)$ and $N_{\delta, k}(r)$. In the sequel, we also derive some convexity properties of these means and also study their relative growths. We shall assume throughout that $\varrho<\infty$.

\section{We now prove}

Lemma. For every $r, 0<r<R,\left[x^{k} I_{\delta}(r) /(R-r)\right]$ is an increasing convex function of $\left[x^{k} N_{\delta, k}(r)\right]$.

Proof. From (1.5) we have

$$
\frac{d\left[x^{k} I_{\delta}(r) /(R-r)\right]}{d\left[x^{k} N_{\delta, k}(r)\right]}=\frac{r I_{\delta}^{\prime}(r)}{R I_{\delta}(r)}+\frac{r}{R(R-r)}+\frac{k}{R-r},
$$

where $I_{\delta}^{\prime}(r)$ denotes the derivative of $I_{\delta}(r)$ with respect to $r$. Since $R$ and $k$ are fixed, the last two terms on the right hand side of the above equation are increasing functions of $r$. Further, it is well known that $\log I_{\delta}(r)$ is an increasing convex function of $\log r$. Hence the right hand side of the above equation is an increasing function of $r$ and the Lemma follows.

Theorem 1. For $\varphi(r)=I_{\delta}(r), J_{\delta}(r)$ and $N_{\delta, k}(r)$, we have

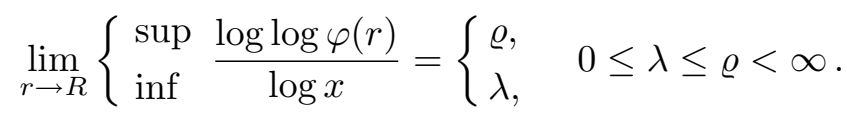

Proof. It is known that for $n \geq 0$,

$$
a_{n}=\frac{1}{2 \pi i} \oint_{C} \frac{f(z)}{z^{n+1}} d z,
$$

where $C$ is the circle $|z|=r, 0<r<R$. Hence

$$
\left|a_{n}\right| r^{n} \leq \frac{1}{2 \pi} \int_{0}^{2 \pi}\left|f\left(r e^{i \theta}\right)\right| d \theta .
$$

Since the right hand side is independent of $n$, we can choose $n$ suitably to obtain

$$
m(r) \leq \frac{1}{2 \pi} \int_{0}^{2 \pi}\left|f\left(r e^{i \theta}\right)\right| d \theta
$$


For $\delta \geq 1$, we apply Hölder's inequality to the right hand side. Then

$$
\begin{aligned}
m(r) & \leq \frac{1}{2 \pi}\left\{\int_{0}^{2 \pi}\left|f\left(r e^{i \theta}\right)\right|^{\delta} d \theta\right\}^{1 / \delta}\left\{\int_{0}^{2 \pi} d \theta\right\}^{(\delta-1) / \delta} \\
& =\left[\frac{1}{2 \pi} \int_{0}^{2 \pi}\left|f\left(r e^{i \theta}\right)\right|^{\delta} d \theta\right]^{1 / \delta} .
\end{aligned}
$$

Hence $m(r) \leq I_{\delta}(r)$. From (1.4) we obviously have $I_{\delta}(r) \leq M(r)$. Hence for $r>0$ and $\delta \geq 1$, we have

$$
m(r) \leq I_{\delta}(r) \leq M(r) .
$$

If $0<\delta<1$, then

$$
\begin{aligned}
2 \pi\left[I_{1+\delta}(r)\right]^{1+\delta} & =\int_{0}^{2 \pi}\left|f\left(r e^{i \theta}\right)\right|^{1+\delta} d \theta \leq M(r) \int_{0}^{2 \pi}\left|f\left(r e^{i \theta}\right)\right|^{\delta} d \theta \\
& =2 \pi M(r)\left[I_{\delta}(r)\right]^{\delta} \leq 2 \pi[M(r)]^{1+\delta}
\end{aligned}
$$

Thus

$$
I_{1+\delta}(r) \leq[M(r)]^{1 /(1+\delta)}\left[I_{\delta}(r)\right]^{\delta /(1+\delta)} \leq M(r) .
$$

From (2.2) we have, in view of (1.3),

$$
\log I_{\delta}(r) \simeq \log M(r) \quad \text { as } r \rightarrow R, \delta \geq 1 .
$$

Hence $\log I_{(1+\delta)}(r) \simeq \log M(r)$ as $r \rightarrow R, 0<\delta<1$. Thus from (2.3) we have

$$
\log I_{\delta}(r) \simeq \log M(r) \quad \text { as } r \rightarrow R, 0<\delta<1 .
$$

Combining these two asymptotic relations, we get

$$
\log I_{\delta}(r) \simeq \log M(r) \quad \text { as } r \rightarrow R, \delta>0 .
$$

From (1.4) and (2.4) we immediately have

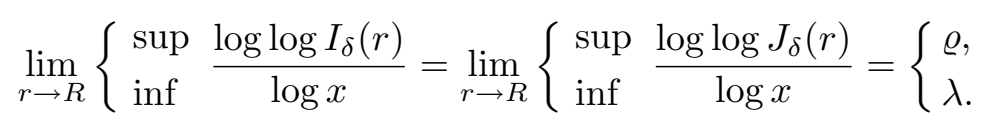

To prove (2.1) for $\varphi(r)=N_{\delta, k}(r)$, we take

$$
r^{\prime}=R\left[1-\frac{1}{\alpha}\left(1-\frac{r}{R}\right)\right]
$$

where $\alpha>1$ is an arbitrary constant. Then from (1.5) we have

$$
\begin{aligned}
N_{\delta, k}\left(r^{\prime}\right) & =\left(x^{\prime}\right)^{-k} \int_{0}^{r^{\prime}} I_{\delta}(y)\left(\frac{R y}{R-y}\right)^{k+1} \frac{d y}{y^{2}} \\
& >\left(x^{\prime}\right)^{-k} \int_{r}^{r^{\prime}} I_{\delta}(y)\left(\frac{R y}{R-y}\right)^{k+1} \frac{d y}{y^{2}},
\end{aligned}
$$


where $x^{\prime}=R r^{\prime} /\left(R-r^{\prime}\right)$. Since $I_{\delta}(r)$ is an increasing function of $r$, we have

$$
N_{\delta, k}\left(r^{\prime}\right)>\frac{I_{\delta}(r)}{k} \frac{\left(x^{\prime}\right)^{k}-x^{k}}{\left(x^{\prime}\right)^{k}}=O(1) I_{\delta}(r) .
$$

It can be easily verified that $x^{\prime} / x \rightarrow \alpha$ and $\left(\log x^{\prime}\right) / \log x \rightarrow 1$ as $r \rightarrow R$. Hence we have

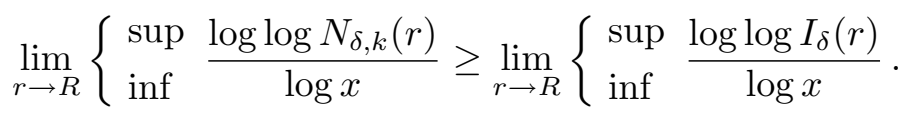

For the reverse inequality we have from (1.5),

$$
N_{\delta, k}(r) \leq I_{\delta}(r) / k \text {. }
$$

Hence

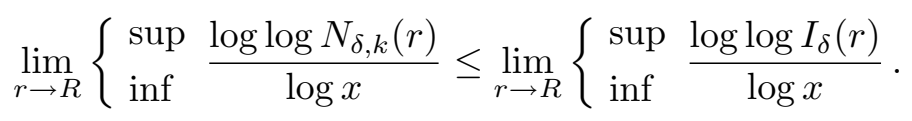

Combining (2.6) and (2.8) we get the relation (2.1) for $\varphi(r)=N_{\delta, k}(r)$. This proves (2.1) completely.

Theorem 2. For $0<\varrho<\infty$, we have

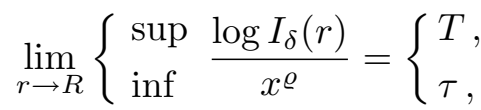

$$
\begin{aligned}
& \lim _{r \rightarrow R}\left\{\begin{array}{l}
\sup \frac{\log N_{\delta, k}(r)}{x^{\varrho}}=\left\{\begin{array}{l}
T, \\
\inf
\end{array} .\right.
\end{array}\right.
\end{aligned}
$$

Proof. The relation (2.9) follows easily from (2.4) and the definitions of $T$ and $\tau$. To prove (2.10) we have from (2.7),

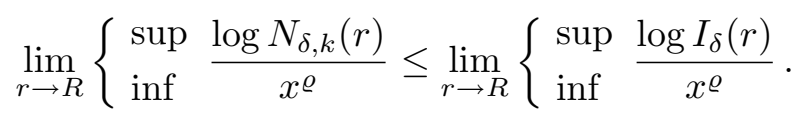

Also, from (2.5) we have

$$
\log N_{\delta, k}\left(r^{\prime}\right)>O(1)+\log I_{\delta}(r) .
$$

Since $x^{\prime} / x \rightarrow \alpha$ as $r \rightarrow R$, we have

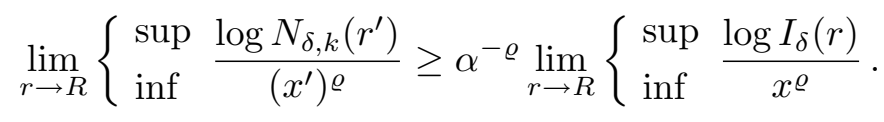

Since $\alpha>1$ was arbitrary, we thus have

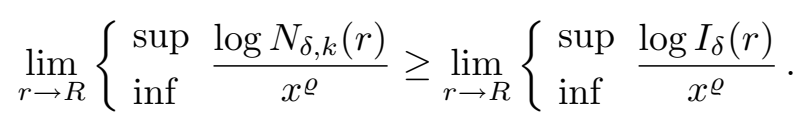

Now combining (2.11) and (2.12), we get (2.10) in view of (2.9). This proves Theorem 2. 
In the next two theorems, we obtain the relative growth of $I_{\delta}(r)$ and $N_{\delta, k}(r)$. We prove

TheOREM 3. For the mean values $I_{\delta}(r)$ and $N_{\delta, k}(r)$ as defined before, we have

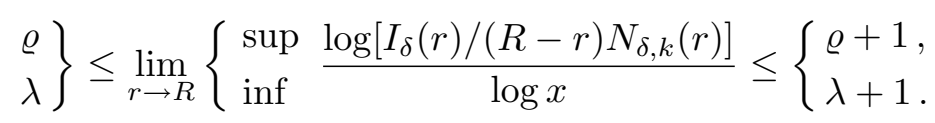

Proof. From (1.5) we have

$$
\frac{d}{d r}\left[x^{r} N_{\delta, k}(r)\right]=x^{k+1} I_{\delta}(r) / r^{2}
$$

where $x=R r /(R-r)$. Expanding and rearranging the terms on the left hand side, we get

$$
\frac{N_{\delta, k}^{\prime}(r)}{N_{\delta, k}(r)}=\frac{R I_{\delta}(r)}{r(R-r) N_{\delta, k}(r)}-\frac{k R}{r(R-r)} .
$$

Integrating on both sides of this equation with respect to $r$, we get

$$
\log N_{\delta, k}(r)=O(1)+R \int_{r_{0}}^{r} \frac{I_{\delta}(y) d y}{y(R-y) N_{\delta, k}(y)}-k \log [r /(R-r)]
$$

where $0<r_{0} \leq r<R$. Since $\varrho<\infty$, we have from Theorem 1 ,

$$
\lim _{r \rightarrow R} \frac{\log (R-r)}{\log N_{\delta, k}(r)}=0 .
$$

Now from the Lemma, $\left[I_{\delta}(y) /(R-y) N_{\delta, k}(y)\right]$ is an increasing function of $y$. Hence from (2.14) we have

$$
\log N_{\delta, k}(r)<O(1)+\frac{R I_{\delta}(r) \log \left(r / r_{0}\right)}{(R-r) N_{\delta, k}(r)}-k \log [r /(R-r)],
$$

or, in view of (2.15),

$$
\log N_{\delta, k}(r)\{1+o(1)\}<\frac{R I_{\delta}(r) \log \left(r / r_{0}\right)}{(R-r) N_{\delta, k}(r)} .
$$

Hence

$$
\lim _{r \rightarrow R}\left\{\begin{array}{l}
\sup \\
\inf
\end{array} \frac{\log \log N_{\delta, k}(r)}{\log x} \leq \lim _{r \rightarrow R}\left\{\begin{array}{l}
\sup \\
\inf \frac{\log \left[I_{\delta}(r) /(R-r) N_{\delta, k}(r)\right]}{\log x} .
\end{array}\right.\right.
$$

In view of (2.1), we get the left hand inequalities of (2.13). To obtain the right hand inequalities of (2.13), we again take arbitrary $\alpha>1$ and 
$r^{\prime}=R[1-(1 / \alpha)(1-r / R)]$. Then from $(2.14)$, since $r^{\prime}>r$,

$$
\begin{aligned}
\log N_{\delta, k}\left(r^{\prime}\right) & \geq O(1)+R \int_{r}^{r^{\prime}} \frac{I_{\delta}(y) d y}{y(R-y) N_{\delta, k}(y)}-k \log \left[r^{\prime} /\left(R-r^{\prime}\right)\right] \\
& \geq O(1)+\frac{R I_{\delta}(r) \log \left(r^{\prime} / r\right)}{(R-r) N_{\delta, k}(r)}-k \log \left[r^{\prime} /\left(R-r^{\prime}\right)\right] .
\end{aligned}
$$

Using (2.15) we have

$$
[1+o(1)] \log N_{\delta, k}\left(r^{\prime}\right) \geq \frac{R I_{\delta}(r) \log \left(r^{\prime} / r\right)}{(R-r) N_{\delta, k}(r)}+O(1),
$$

or

$$
\frac{\log \log N_{\delta, k}\left(r^{\prime}\right)}{\log x} \geq \frac{\log \left[I_{\delta}(r) /(R-r) N_{\delta, k}(r)\right]}{\log x}+\frac{\log \log \left(r^{\prime} / r\right)}{\log x}+o(1) .
$$

As before, $(\log x) / \log x^{\prime} \rightarrow 1$ and $\left[\log \log \left(r^{\prime} / r\right)\right] / \log x \rightarrow-1$ as $r \rightarrow R$.

Hence we obtain, on proceeding to limits,

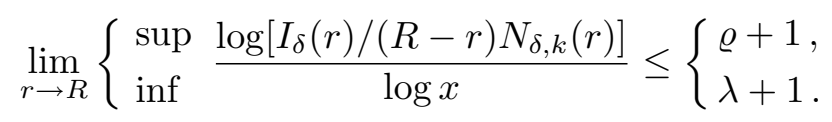

This proves Theorem 3 .

TheOrem 4. For $0<\varrho<\infty$, we have

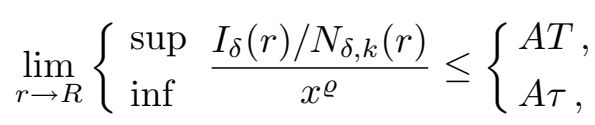

where $A=(\varrho+1)^{\varrho+1} / \varrho^{\varrho}$.

Proof. From (2.16) we have

$$
[1+o(1)] \frac{\log N_{\delta, k}\left(r^{\prime}\right)}{\left(x^{\prime}\right)^{\varrho}} \geq \frac{R \log \left(r^{\prime} / r\right) I_{\delta}(r)}{(R-r) N_{\delta, k}(r)\left(x^{\prime}\right)^{\varrho}}+o(1) .
$$

Since

$$
\lim _{r \rightarrow R} \frac{\log \left(r^{\prime} / r\right)}{R-r}=\frac{\alpha-1}{\alpha R} \text { and } \lim _{r \rightarrow R} \frac{x^{\prime}}{x}=\alpha,
$$

where as before $x^{\prime}=R r^{\prime} /\left(R-r^{\prime}\right)$, we get on proceeding to limits

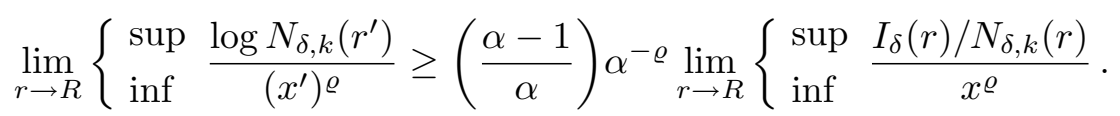

Since $\alpha>1$ was arbitrary, we can take $\alpha=(\varrho+1) / \varrho$. Hence, using $(2.10)$ we obtain

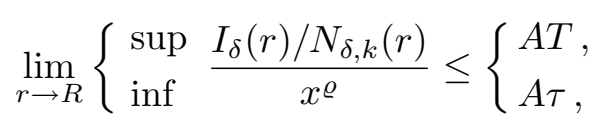

where $A=(\varrho+1)^{\varrho+1} / \varrho^{\varrho}$. Thus Theorem 4 follows. 
The authors are thankful to the referee for his valuable comments and suggestions.

\section{References}

[1] R. P. Boas, Entire Functions, Academic Press, New York 1954.

[2] G. H. Hardy, The mean value of the modulus of an analytic function, Proc. London Math. Soc. 14 (2) (1915), 269-277.

[3] G. P. Kapoor, A note on the proximate order of functions analytic in the unit disc, Rev. Fac. Sci. Univ. d'Istanbul Sér. A 36 (1971), 35-40.

[4] L. R. Sons, Regularity of growth and gaps, J. Math. Anal. Appl. 24 (1968), 296-306.

DEPARTMENT OF MATHEMATICS

UNIVERSITY OF ROORKEE

ROORKEE 247667, INDIA

Reçu par la Rédaction le 30.4.1991 